\title{
„Das Leben ist nur ein Spiegel“ - Schopenhauers kritischer Lebensbegriff
}

\author{
von Matthias Koßler \\ Johannes-Gutenberg-Universität-Mainz (Deutschland)
}

\begin{abstract}
ZUSAMMENFASSUNG
Schopenhauer gilt als Vorreiter oder erster Vertreter der Lebensphilosophie, wie sie später von Dilthey, Nietzsche, Bergson und anderen entfaltet wurde. Im Unterschied zur klassischen Lebensphilosophie und $\mathrm{zu}$ den an sie anknüpfenden ethischen Entwürfen wird das Leben bei Schopenhauer aber nicht als Zweck betrachtet, sondern als Mittel zu einem anderen Zweck, nämlich für die Ethik, die in der Verneinung des Willens zum Leben gipfelt. Schon in den frühesten handschriftlichen Aufzeichnungen verwendet Schopenhauer verschiedene Metaphern, um das Leben als Medium der ethischen Erkenntnis zu charakterisieren. Besonders häufig tritt dabei das Bild vom Leben als Spiegel auf, in dem der Mensch sein eigenes Wesen und damit auch das Wesen der Welt erblickt. Im Hauptwerk wird dann dieses Bild vom Begriff der Objektivation des Willens abgelöst. Insofern die höchste und deutlichste Objektivation des Willens der Lebenslauf des menschlichen Individuums darstellt, ist Schopenhauers Lebensbegriff eng mit seiner Charakterlehre verknüpft. In dem Beitrag wird das verwickelte Verhältnis von Leben, Erkennen und Ethik vor dem Hintergrund der proto-hermeneutischen Metaphysikkonzeption Schopenhauers anhand der Entwicklung der Spiegelmetapher betrachtet.
\end{abstract}

\section{ABSTRACT}

Schopenhauer is said to be precursor or first exponent of the philosophy of life as it has been developed later on by Dilthey, Nietzsche, Bergson and others. However, in contrast to classical philosophy of life and to the ethical conceptions going back to it, in Schopenhauer life is not viewed as an end but as a means to another end, namely to the ethics which culminates in the denial of the will-to-live. Already in the earliest hand-written manuscripts Schopenhauer uses different metaphors in order to characterize life as a medium of ethical knowledge. In particular the image of life as a mirror in which a human being sees his own nature and thus realizes the essence of the world often occurs. In the mature work this image is replaced by the concept of objectivation of will. As far as the highest and clearest objectivation of will is the life story of a human individual Schopenhauer's concept of life is bound up with his theory of character. In my essay I consider the complicated relationship between life, knowledge and ethics in Schopenhauer on the background of his proto-hermeneutic metaphysics by following the development of his use of the mirror-metaphor.

„Das Leben ist eine mißliche Sache. Ich habe mir vorgesetzt, es damit

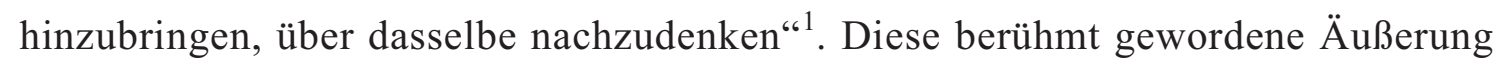
des 23-jährigen Schopenhauer gegenüber Martin Wieland umreißt bereits seine besondere Haltung gegenüber dem Leben, das in seiner Nachfolge zu einem so

\footnotetext{
${ }^{1}$ Arthur Schopenhauer: Gespräche (ed. Arthur Hübscher). Stuttgart / Bad Canstatt 1971, S. 22.
} 
wichtigen Begriff der Philosophie wurde, daß man von einer, vor allem durch Dilthey, Nietzsche und Bergson entwickelten „Lebensphilosophie“ spricht. Während dort das Leben als begrifflich unzugängliche aber sinnstiftende Instanz und in ethischer Hinsicht als Zweck betrachtet wird, ist der Lebensbegriff bei Schopenhauer in dem Sinne problematisch, daß das Leben aus sich heraus keinen Sinn hat und nicht als Zweck an sich selbst betrachtet werden kann. Vielmehr ist es ein Mittel zu einem anderen Zweck, und es erfordert begriffliche Überlegung, den Sinn des Lebens als Mittel zum Zweck aufzudecken.

Diese Überlegung, das Nachdenken über das Leben, ist die genuin philosophische Tätigkeit. Wie Schopenhauer in den bekannten Ausführungen über das metaphysische Bedürfnis des Menschen darlegt, zeichnet sich der Mensch gegenüber allen anderen Wesen dadurch aus, daß sich ihm das Leben nicht von selbst versteht, sondern zu einem „Problem“ wird, „dessen sogar der Roheste und Beschränkteste, in einzelnen helleren Augenblicken. lebhaft inne wird“². Die Empfindung der Rätselhaftigkeit des Daseins ist mit der Besinnung auf den Tod und das Übel in der Welt verknüpft. Denn das abstrakte Bewußtsein des Todes stellt die Selbstverständlichkeit des Lebens sowie die Annahme der Welt und des Daseyns als eines „schlechthin nothwendigen Wesens“ in Frage ${ }^{3}$. Die Welt und das Leben könnten auch nicht sein, und damit stellt sich die Frage, wozu sie überhaupt sind. Diese Frage wird um so drängender, je deutlicher sich das Bewußtsein vom Übel in der Welt hinzugesellt. Denn dadurch steht nicht mehr nur die Möglichkeit der Nichtexistenz der Welt im Raum, sondern angesichts des Bösen und des Übels drängt sich die Forderung auf, daß die Welt und das Leben „Etwas sind, was ganz und gar und überhaupt nicht sein sollte“4 . Daher spricht Schopenhauer vom Tod als dem „Musaget“ der Philosophie ${ }^{5}$; das „Böse, das Übel und der Tod sind es, welche das philosophische Erstaunen qualifizieren und erhöhen“66.

Man muß in diesem Zusammenhang die unter dem Etikett „Pessimismus“ firmierende Betonung des Leidens und der Nichtigkeit des Lebens bei Schopenhauer vor dem Hintergrund seiner Zeit sehen, in der der Glaube an den technischen und

\footnotetext{
${ }^{2}$ Arthur Schopenhauer: Die Welt als Wille und Vorstellung, Bd. II (ZA Bd. 3), Kap. 17, S. 200. Schopenhauers Werke werden zitiert nach der Ausgabe: Arthur Schopenhauer, Werke in zehn Bänden. Zürcher Ausgabe (=ZA). Zürich 1977.

${ }^{3}$ Ibid., S. 199. Vgl. Clement Rosset, Schopenhauer, philosophe de l'absurde, Paris 1967, S. 13 ff.

${ }^{4}$ Die Welt als Wille und Vorstellung, Bd. II (ZA Bd. 3), Kap. 17, S. 200 (Hervorhebung von mir, M. K.)

${ }^{5}$ Die Welt als Wille und Vorstellung, Bd. II (ZA Bd. 4), Kap. 41, S. 543.

${ }^{6}$ Die Welt als Wille und Vorstellung, Bd. II (ZA Bd. 3), Kap. 17, S. 201.
}

ethic@-Florianópolis, v. 11, n. 2, p.1-15,julho de 2012. 
wissenschaftlichen Fortschritt den Gedanken des „Rätsels“ zugunsten der Rede von zu erledigenden Aufgaben aus den Wissenschaften und der Philosophie zu verdrängen begann. Gerade in bezug auf das Leben sind heute die Aufgaben der Erhaltung, der Verlängerung und der Verbesserung in aller Munde, während die Frage nach dem Sinn dessen, was da erhalten, verlängert und verbessert werden soll, nicht nur nicht gestellt wird, sondern auch als moralisch anrüchig gilt. Das ist auch durchaus berechtigt, weil wir derart in der Haltung der Machbarkeit verhaftet sind, daß die Rede von der Wertlosigkeit des Lebens sogleich als Aufforderung verstanden wird, Abgrenzungen von wertem und unwertem Leben vorzunehmen und Maßnahmen zur Verkürzung des letzteren einzuleiten. Ein Rätsel ist aber etwas anderes als eine Aufgabe, zu deren Erledigung die Mittel schon feststehen; und die Philosophie strebt nicht nach einer wissenschaftlichen Erklärung, sondern nach einer Erhellung der von jedem irgendwann einmal empfundenen Rätselhaftigkeit unseres Daseins: „,.. wenn irgend etwas auf der Welt wünschenswerth ist, so wünschenswerth, daß selbst der rohe und dumpfe Haufen, in seinen besonneneren Augenblicken, es höher schätzen würde, als Silber und Gold; so ist es, daß ein Lichtstrahl fiele auf das Dunkel unsers Daseyns und irgend ein Aufschluß uns würde über diese räthselhafte Existenz, an der nichts klar ist, als ihr Elend und ihre Nichtigkeit ${ }^{67}$.

Wenn das Leben selbst zum Problem wird - und das wird es nach Schopenhauer zwangsläufig -, dann kann die Lösung des Problems nicht im Bereich der Erfahrung zu suchen sein. Philosophie im Schopenhauerschen Verständnis des Nachdenkens über das Rätsel der Welt muß Metaphysik sein. Metaphysik ist für Schopenhauer nicht, wie Nietzsche meinte, ein Überbleibsel der philosophischen Tradition, ein „Nachschössling ${ }^{68}$ der Religion, sondern macht den „noch nie dagewesenen Gedanken“9 aus, den er für seine Lehre reklamiert. Denn ihm ist durchaus klar, daß der Bereich der Erfahrung nicht wie in der traditionellen, vorkritischen Metaphysik durch Schlüsse auf transzendente Gründe überschritten werden kann. An die Stelle der nach dem Satz vom Grunde verfahrenden traditionellen Metaphysik setzt er seine Konzeption einer „,immanenten“ Metaphysik, die darauf beruht, daß der Wille „den Schlüssel zum Räthsel der Welt" ${ }^{\text {10 }}$ bietet. „Immanente Metaphysik“ bedeutet nicht die Erklärung der

\footnotetext{
${ }^{7}$ Ibid., S. 191.

${ }^{8}$ Friedrich Nietzsche: Die fröhliche Wissenschaft, in: Werke. Kritische Gesamtausgabe (KGW) (ed. G. Colli / M. Montinari ) Bd. V, 2, Berlin / New York 1973, S. 11-335, hier S. 172 (Nr. 151)

${ }^{9}$ Die Welt als Wille und Vorstellung, Bd. II (ZA Bd. 3), Kap. 17, S. 215.

${ }^{10}$ Die Welt als Wille und Vorstellung, Bd. II (ZA Bd. 3), Kap. 17, S. 209, (ZA Bd. 4), Kap. 50, S. 750.

ethic@-Florianópolis, v. 11,n. 2, p. 1-15, julho de 2012.
} 
Welt durch die vorgebliche Erkenntnis eines außerhalb der Erfahrung Liegenden, sondern die „Entzifferung“ der Welt mithilfe der inneren Erfahrung in der Art und Weise, in der eine Geheimschrift durch den richtigen Code dechiffriert wird ${ }^{11}$. Der metaphysische Begriff des Willens, den Schopenhauer eher mißverständlich als „Ding an sich“ bezeichnet, hat so seine Berechtigung nur insofern und so lange, als mit ihm die Erfahrung als ein Ganzes ,gedeutet, ausgelegt“ werden kann ${ }^{12}$. Nur wenn man diese hermeneutische Konzeption von Metaphysik berücksichtigt, ergeben die auf den ersten Blick kontradiktorischen Formulierungen wie die, daß der Wille „blos relativ, d.h. in seinem Verhältnis zur Erscheinung“ Ding an sich ist ${ }^{13}$, einen Sinn; und nur so wird verständlich, daß Schopenhauer einerseits den Willen als Ding an sich bezeichnet, andererseits aber im selben Atemzug betont, daß die Frage nach dem Ding an sich, unabhängig davon, daß es als Wille erscheint, ,,nie zu beantworten“ $\operatorname{sei}^{14}$.

Um wieder auf die Problematik des Lebens zurückzukommen, so ist also der Sinn und Zweck des Lebens weder in ihm selbst, wie es sich unmittelbar darbietet, noch in einem Jenseitigen $\mathrm{zu}$ suchen, sondern in einer Deutung der Erscheinungen des Lebens, durch die sie ein zusammenhängendes Ganzes ausmachen. In der reifen Philosophie Schopenhauers ist der Schlüssel zu einer solchen Deutung der Wille. Unter der Annahme, daß das Leben eine Äußerung des Willens vor dem Hintergrund der Möglichkeit seiner Bejahung und Verneinung ist, soll die Erfahrung des Elends und der Nichtigkeit des Lebens einen Sinn ergeben. $\mathrm{Zu}$ dieser reifen Lehre gelangt Schopenhauer durch eine Entwicklung von Gedanken über das Leben, die er in verschiedenen Metaphern zu fassen sucht. Diese Entwicklung, die im Spannungsfeld zwischen Religion und Nihilismus, zwischen einer Heilslehre und einer Philosophie des Absurden verläuft, soll im folgenden kurz umrissen werden.

Den Ausgangspunkt bildet die Fragwürdigkeit des Lebens als solchen, die, wie gezeigt, für Schopenhauer das Bedürfnis der Metaphysik hervorruft und in seinen frühesten handschriftlichen Aufzeichnungen schon deutlich zum Ausdruck kommt. Dort ist noch eine stark religiöse Komponente im Nachdenken über das Leben enthalten. Von der später entwickelten Auffassung der Religion als „Volksmetaphysik“"15 her gesehen, liegt das auf der Hand, haben doch die Religionen wie die Philosophie die Funktion, das

\footnotetext{
${ }^{11}$ Die Welt als Wille und Vorstellung, Bd. II (ZA Bd. 3), Kap. 17, S. 213.

12 Ibid.

${ }^{13}$ Arthur Schopenhauer: Gesammelte Briefe (ed. A. Hübscher). Bonn 21987, S. 291.

${ }^{14}$ Die Welt als Wille und Vorstellung, Bd. II (ZA Bd. 3), Kap. 18, S. 231.

${ }^{15}$ Die Welt als Wille und Vorstellung, Bd. II (ZA Bd. 3), Kap. 17, S. 192.
}

ethic@-Florianópolis, v. 11,n. 2, p. 1-15, julho de 2012. 
Bedürfnis nach einer metaphysischen Dimension des Lebens zu stillen. Nahezu alle Religionen tun dies dadurch, daß sie Aussicht auf ein Dasein jenseits des in der Erfahrung sich darstellenden Lebens eröffnen, durch die das gegenwärtige Leben seinen Sinn erst erhält. Das Judentum sieht Schopenhauer deshalb als ,die roheste und schlechteste aller Religionen“" an, weil sie kein Dasein nach dem Tode kenne und daher „eine Religion ohne alle metaphysische Tendenz“"16 d.h. eigentlich gar keine Religion im Schopenhauerschen Verständnis sei. Im Christentum dagegen ist der Gedanke geläufig, daß das Leben vor dem Tode nur eine Vorbereitung auf das ewige Leben ist, oder eine Pilgerreise in die Fremde in der sich der Christ bewähren muß. Der Tod erweist sich so als Übergang vom ,alten“, „fleischlichen“ Leben zum „neuen“, „geistigen“, wobei sich die Bedeutung des alten Lebens als moralische Prüfung erweist.

An die Gegenüberstellung von fleischlichem und geistigem Leben knüpft der junge Schopenhauer an, wobei schon von Anfang an das geistige Leben nicht nur in der Religion, sondern auch in der Kunst und der Ethik gesehen wird. Die früheste einschlägige Notiz von 1809 (dem Jahr, in dem er sein Studium in Göttingen aufnimmt), nennt diese drei Bereiche, die dem Leben Bedeutung verleihen: „Nehmen wir aus dem Leben die wenigen Augenblicke der Religion, der Kunst und der reinen Liebe, was bleibt als eine Reihe trivialer Gedanken?“17, und kurz darauf wird das Leben

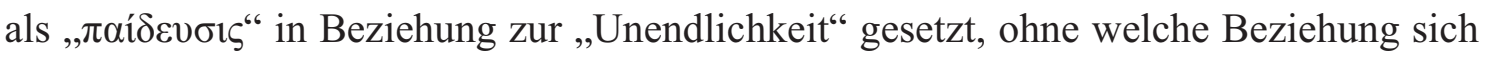
die Frage stelle „wozu das Possenspiel der Welt?““18. Der Gedanke vom Leben als Erziehung, als Lehre wird im folgenden Jahr in einer Weise zugespitzt, die das Leben ganz auf diese Funktion reduziert: „Das Leben ist eine Sprache, in der uns eine Lehre gegeben wird. Könnte diese Lehre uns auf eine andere Weise beigebracht werden, so lebten wir nicht ${ }^{\star 19}$. Diese ersten Reflexionen über das Leben haben noch eine religiöse Basis, indem sie ein „überzeitliches Seyn“, das „Übersinnliche“ bzw. das „ReichGottes“ voraussetzen, dem gegenüber das Leben auch als „eine Nacht, die ein langer Traum füllt" bezeichnet wird ${ }^{20}$. Aus ihnen geht ab 1812 die sogenannte Lehre vom „besseren Bewußtseyn“ hervor, das dem zeitlichen Bewußtsein, also dem sinnlichen Leben entgegengesetzt ist und eben in den erwähnten Erscheinungen der Religion, der Kunst und der Liebe zum Ausdruck kommt. Mit dem ersten Auftreten des „besseren

\footnotetext{
${ }^{16}$ Arthur Schopenhauer: Parerga und Paralipomena, Bd. I (ZA Bd. 7), S. 143 ff., Anm.

${ }^{17}$ HN, Bd. I, S. 10. Schopenhauers nachgelassene Schriften werden zitiert nach der Ausgabe: Arthur Schopenhauer: Der Handschriftliche Nachlaß (Hrsg. Arthur Hübscher), 5 Bde. München 1985 (= HN)

18 Ibid.

${ }^{19}$ HN, Bd. I, S. 13.

${ }^{20}$ HN, Bd. I, S. 15, 20.
}

ethic@-Florianópolis, v. 11, n. 2, p. 1-15, julho de 2012. 
Bewußtseyns“ in den Handschriften ${ }^{21}$ fällt zeitlich auch Schopenhauers Abkehr von der Religion zusammen, die er aufgrund der Aufklärung an ihrem Ende sieht ${ }^{22}$. Damit stellt sich aber die Frage, wie das bessere Bewußtsein als ein Jenseits des zeitlichen, mit dem es nicht „,zu vermitteln und zu verbinden“ ist ${ }^{23}$, ohne religiöse Annahmen bestimmt werden kann. Wenn es kein Leben nach dem Tode gibt, wie es nur die Religionen behaupten können, wie kann dann das Leben eine Lehre für einen anderen Zweck sein?

Es ist daher konsequent, wenn Schopenhauer feststellt, daß wir von dem besseren Bewußtsein „nur negativ sprechen“ können ${ }^{24}$. Dem empirischen, zeitlichen Bewußtsein ist das bessere Bewußtsein bloß ,gänzlicher Untergang, Tod und Vernichtung ${ }^{625}$. So bleibt auch für die Frage nach der Bedeutung des Lebens nur die negative Auskunft, daß das bessere Bewußtsein „im Leben, nicht das Leben will ${ }^{\text {“26. }}$ Diese asketisch-mystische Auffassung von der Bedeutung des Lebens bleibt bekanntlich auch im Hauptwerk erhalten und bestimmt die Form, in der Schopenhauer seine Lehre von der Verneinung des Willens zum Leben präsentiert. Die bloß negative Bestimmung eines Jenseits des Lebens ist allerdings sehr problematisch, scheint sie doch wieder auf die absolute Nichtigkeit des Lebens hinauszulaufen. Schopenhauer versucht dieser Gefahr des Nihilismus logisch-immanent entgegenzutreten, indem er die Denkbarkeit eines absoluten Nichts oder nihil negativum überhaupt bestreitet: „So wird also jedes nihil negativum, oder absolute Nichts, wenn einem höhern Begriff untergeordnet, als ein bloßes nihil privativum, oder relatives Nichts, erscheinen, welches auch immer mit Dem, was es negirt, die Zeichen vertauschen kann, so daß dann jenes als Negation, es selbst aber als Position gedacht würde“ ${ }^{، 27}$. Bereits in einer Aufzeichnung von 1812 hatte Schopenhauer diesen Gedanken in deutlicher Anspielung auf die Lehre vom besseren Bewußtsein auf das Leben bezogen: Mit Recht - so heißt es dort - sagen wir „'uns, als Lebenden, ist Leben und Seyn Eins, Tod und Nichtseyn auch Eins.' - Dagegen, wenn wir uns unserer bewußt werden als nicht in Zeit und Raum, - dann nennen wir eben das was in diesen ist, mit recht Nichts ${ }^{628}$.

Der Grund für die Annahme eines nihil privativum, der „höhere Begriff“, dem das Nichts untergeordnet wird, fehlt in der Lehre vom besseren Bewußtsein. Es muß ein

\footnotetext{
${ }^{21} \mathrm{HN}$, Bd. I, S. 23.

${ }^{22}$ HN, Bd. I, S. 20.

${ }^{23} \mathrm{HN}, \mathrm{Bd}$. I, S. 111

${ }^{24} \mathrm{HN}$, Bd. I, S. 23.

${ }^{25}$ HN, Bd. I, S. 79.

${ }^{26}$ HN, Bd. I, S. 102.

${ }^{27}$ Die Welt als Wille und Vorstellung, Bd. I (ZA Bd. 2), § 71, S. 505.

${ }^{28} \mathrm{HN}$, Bd. I, S. 35.
} 
höherer Begriff als das Dasein, also ein die Erfahrung übersteigender, transzendenter Begriff sein: der des Dinges an sich. Hier ist der logische Ort für die Forderung nach einer immanenten Metaphysik, um die nihilistische Konsequenz aus dem Verfall der Religion zu vermeiden. Die Konzeption des besseren Bewußtseins schwankt nur zwischen einem religiösen Verständnis eines „,heiligen Willens“ und dem nihilistischen eines „Uebergangs ins Nichts“629 hin und her - ein Hin- und Herschwanken, das sich im vierten Buch des Hauptwerks über die Analogie zur Gnadenwirkung durchhält bis zu dem auf den „gravierendsten Gedankenstrich der Philosophie ${ }^{\text {‘30 }}$ folgenden letzten Wort „Nichts“, das Schopenhauer in seinem Handexemplar noch einmal mit einer Fußnote über das „Pradschna-Paramita der Buddhaisten“31 versah. Inzwischen jedoch hatte er sein Konzept einer immanenten Metaphysik entwickelt, die auf einer eigentümlichen Verwendung des von Kant übernommenen Begriffs des Dinges an sich beruht. „Die Brücke also", schreibt Schopenhauer im Kapitel Ueber das metaphysische Bedürfniß des Menschen, ,auf welcher die Metaphysik über die Erfahrung hinausgelangt, ist nichts Anderes, als eben jene Zerlegung der Erfahrung in Erscheinung und Ding an sich, worin ich Kants größtes Verdienst gesetzt habe. Denn sie enthält die Nachweisung eines von der Erscheinung verschiedenen Kernes derselben“, der ,jedoch immer nur als das in ihre Erscheinende, nicht aber unabhängig von aller Erscheinung“ betrachtet wird ${ }^{32}$.

Es ist für das Verständnis dieser „,immanenten“ Verwendung des Begriffes vom Ding an sich - das hier ja nicht ein die Erfahrung Übersteigendes, sondern nur die Brücke zu einem solchen bezeichnet - wichtig zu realisieren, daß der Ursprung von Schopenhauers Metaphysik nicht in Kants Lehre vom Ding an sich zu finden ist, sondern in dessen Unterscheidung zwischen intelligiblem und empirischem Charakter. In Schopenhauers Studienheften zu Kant aus der Göttinger Zeit finden sich nur polemische Anmerkungen zum Ding an sich, das er als „die schwache Seite der K[anti]schen Lehre“ bezeichnet ${ }^{33}$. Und noch in der mit dem Titel Gegen Kant versehenen Sammlung von Notizen aus dem Jahr 1816 wird im Unterschied zum Anhang des Hauptwerks der kantische Begriff des Dinges an sich durchaus kritisch gesehen. Dagegen lobt er schon hier, wie auch mehrfach später in den veröffentlichten Schriften, überschwenglich die Lehre vom intelligiblen und empirischen Charakter:

\footnotetext{
${ }^{29}$ HN, Bd. I, S. 245.

${ }^{30}$ Vgl. Ludger Lütkehaus: Einleitung zu Arthur Schopenhauers Werke in fünf Bänden (ed. L. Lütkehaus), Beibuch. Frankfurt/M 2006, S. 9-34, hier S. 22.

${ }^{31}$ Die Welt als Wille und Vorstellung, Bd. I (ZA Bd. 2), § 71, S. 508.

${ }^{32}$ Die Welt als Wille und Vorstellung, Bd. II (ZA Bd. 3), Kap. 17, S. 214.

${ }^{33}$ HN, Bd. II, S. 265.
} 
„Was K[ant] bei Gelegenheit der Auflösung dieser 3ten Antinomie über das Verhältniß des Dings an sich zur Erscheinung und des intelligiblen Kar[a]kt[er]s zum empirischen sagt, gehört an sich zum Vortrefflichsten, was je von Menschen gesagt ist ${ }^{\text {‘34 }}$ (HN II 421). Falsch daran sei jedoch, daß Kant mit der Konzeption der Kausalität aus Freiheit im intelligiblen Charakter den Willen als Ding an sich als Ursache seiner Erscheinung gefaßt habe.

\begin{abstract}
Statt dessen hätte K[ant] unmittelbar vom Willen ausgehn[,] ihn als das unmittelbar erkannte Ansich unsrer eignen Erscheinung nachweisen und dann zeigen sollen, wie alle Handlungen, obwohl durch Motive bedingt und verursacht, doch sowohl von ihrem Urheber selbst, als von fremden Beurtheilern, jenem nothwendig und apodiktisch selbst und allein zugeschrieben werden, als lediglich von ihm abhängend, und Schuld und Verdienst ihm danach zuerkannt wird. Dies allein leitet auf die Erkenntniß von dem was nicht Erscheinung [...] ist, sondern nur durch diese Erscheinung sich kundthut, erkennbar wird $[\ldots]^{35}$
\end{abstract}

Die „Brücke“, die über die Erfahrung hinaus führt, beruht also auf der Zuschreibung der empirischen Handlungen eines Menschen zu seinem Wesen, d.h. auf dem Verhältnis von intelligiblem und empirischem Charakter, wie es Schopenhauer drei Jahre zuvor in der Dissertation im Rahmen der Erörterung des Satzes vom Grunde des Handelns dargelegt hatte. Den intelligiblen Charakter führt er dort als einen „beharrlichen Zustand des Subjekts des Wollens“ ein, zu dessen Annahme die Beobachtung leitet, daß verschiedene Menschen bei gleichen gegebenen Motiven unterschiedliche Handlungsweisen zeigen, während derselbe Mensch unter gleichen Umständen immer dieselbe Handlungsweise an den Tag legt. Im intelligiblen Charakter erweist sich, daß das Wollen „seiner Natur nach im höchsten Grade frey, ja das innerste von allem Andern unabhängige Wesen des Menschen selbst ist ${ }^{\star 36}$. Hier taucht zum ersten Mal der Gedanke vom Willen als dem Wesen des Menschen auf, allerdings noch substantiell und in kausaler Relation zum Handeln stehend gedacht. Doch schon bald zieht Schopenhauer Konsequenzen daraus, daß der intelligible Charakter ja nicht eigentlich Ursache ist, sondern die Bedingung dafür, daß eine Ursache, nämlich das Motiv, zur Wirkung kommt. Auch steht er als das Wesen des Menschen nicht unter den

\footnotetext{
${ }^{34}$ HN, Bd. II, S. 421.

${ }^{35}$ Ibid. (Hervorhebung von mir, M. K.)

${ }^{36}$ Arthur Schopenhauer: Über die vierfache Wurzel des Satzes vom zureichenden Grunde, erste Auflage (1813). In: Sämtliche Werke (Hrsg. Arthur Hübscher), Bd. 7, Wiesbaden ${ }^{2}$ 1950, S. 1-94, hier S. 76.
}

ethic@-Florianópolis, v. 11, n. 2, p. 1-15, julho de 2012. 
Bestimmungen des Raumes und der Zeit und kann daher nicht als Beharrendes in der Zeit, als Substanz gefaßt werden.

Bald nach Abschluß der Dissertation tritt daher an die Stelle der Kausalrelation zwischen intelligiblem und empirischem Charakter ein Verhältnis, das durch die Metapher des Spiegels ausgedrückt wird. Zugleich geht nach und nach, im Zuge der Übertragung des Verhältnisses von den menschlichen Handlungen auf andere Erscheinungen, die Rede vom intelligiblen Charakter zu der vom Willen über. Der Wille offenbart sich an den erscheinenden Handlungen. Weder wirkt er auf die äußeren Erscheinungen noch können diese auf ihn - etwa auf eine Änderung seines Charakters einwirken; er wird an ihnen nur sichtbar. Zunächst versucht Schopenhauer, dieses Verhältnis durch verschiedene Metaphern zu fassen: „das Leben ist dem Menschen, d. h. dem Willen, eben das, was die chemischen Reagenzien dem Körper: nur an diesem offenbart sich was er ist und nur sofern er [wohl: es (M. K.)] sich also offenbart ist es"; oder „Das Leben ist der Korrekturbogen daran die im Setzen begangnen Fehler offenbar werden“; und schließlich „Das Leben ist nur der Spiegel, in welchen man sieht nicht damit er abspiegele, sondern damit man sich daran erkenne, sehe, was er spiegelt ${ }^{637}$. Die Spiegelmetapher setzt sich dann in den nachfolgenden Aufzeichnungen durch und wird bis zum Abschluß des Hauptwerks in verschiedenen Nuancierungen durchgespielt.

Bevor die Verwendung der Spiegelmetapher genauer betrachtet wird, ist noch einmal auf die Bedeutung des Umstands zurückzukommen, daß Schopenhauers Lehre vom Willen als Ding an sich ihren Ausgangspunkt nicht beim kantischen Verständnis des Dinges an sich hat, sondern bei der Betrachtung des menschlichen Charakters ansetzt. Es ist demnach nämlich nicht so, als ob Schopenhauer das kantische Ding an sich unter Berufung auf ein unmittelbares Innewerden des blinden Willenstriebs mit einer positiven Bestimmung gefüllt hätte. Der Gedanke eines blinden Willens, der auch die unbewußten Affekte und alle körperlichen Empfindungen bezeichnet, beruht auf einer durch Abstraktion und Reflexion zustande kommenden „Erweiterung des Begriffs“"Wille ${ }^{38}$. Ausgangspunkt ist nicht das Gefühl, von Leidenschaften getrieben zu sein, sondern das Gefühl der Zurechnung von Handlungen zur eigenen Person, zum individuellen Charakter. Die einzelnen, zeitlich erfolgenden Willensakte werden dabei als ein Sichtbarwerden des gestaltlosen inneren Wesens erfahren, des Subjekts, das die in den Handlungen sich äußernden Willensakte auf sich bezieht und verantwortet.

\footnotetext{
${ }^{37} \mathrm{HN}$, Bd. I, S. 91.

${ }^{38}$ Die Welt als Wille und Vorstellung, Bd. I (ZA Bd. 1), § 22, S. 156; vgl. ibid., § 1, S. 30.

ethic@-Florianópolis, v. 11,n. 2,p.1-15, julho de 2012.
} 
Unmittelbar erfahren wird nicht der Wille als Ding an sich, sondern der einzelne Willensakt als Brücke zu diesem an sich selbst unerkennbar bleibenden Wesen. Die Rede vom Willen als Ding an sich beruht auf einer Auslegung dieser Erfahrung des Sichtbarwerdens, das als Prozeß die Handlungen mit ihrem inneren Ursprung verknüpft. Ding an sich und Erscheinung sind hermeneutische Begriffe und daher immer aufeinander bezogen. Darin unterscheidet sich ihre Verwendung bei Schopenhauer von vorneherein grundsätzlich von der bei Kant. Seine Kritik an Kants Anwendung des Kausalitätsverhältnisses auf Ding an sich und Erscheinung hat ihren Grund darin, daß dabei das Ding an sich als gegeben vorausgesetzt und nicht aus der Erscheinung erst gedeutet wird. „Wenn von Ursache und Wirkung geredet wird, darf das Verhältniß des Willens zu seiner Erscheinung (oder des intelligiblen Charakters zum empirischen) nie herbeigezogen werden ..." ${ }^{\text {39 }}$. In der ersten Auflage der Welt als Wille und Vorstellung hatte diese Stelle den später gestrichenen Zusatz: ,ja es kann eigentlich nur gleichnißweise ein Verhältniß genannt werden““40. Denn Verhältnisse bestehen nur zwischen Gegenständen der Erfahrung, nicht zu dem Transzendenten, wo von Verhältnissen nur in auslegender Rede gesprochen werden kann.

Dieses Verhältnis des Offenbarwerdens, das eigentlich kein Verhältnis ist, versucht Schopenhauer mit der Metapher des Spiegels zu erhellen, die, wie alle Metaphern, mehrere Verhältnisarten einschließt. Wenn es in dem schon angeführten Beispiel heißt, daß man in den Spiegel des Lebens sehe, „nicht damit er abspiegele, sondern damit man sich daran erkenne, sehe, was er spiegelt", so ist damit das Leben als Medium der Selbsterkenntnis angesprochen, das gerade keine eigene Bedeutung haben darf, um seine Funktion zu erfüllen. Man soll sozusagen durch das Leben hindurch auf das blicken, was sich in ihm offenbart, so wie man auf die Handlungen eines Menschen achtet, um seinen Charakter kennenzulernen. Die Formulierung „nicht damit er abspiegele“ bedeutet, daß der Spiegel nicht als ein Gegenstand aus Metall und Glas, der die Eigenschaft hat zu spiegeln, betrachtet wird, sondern nur in der Funktion, ein Anderes durch sich sichtbar zu machen. Es unterscheidet das Spiegelbild vom Bild oder Abbild, daß es in dieser Weise auf die bloße Funktion des Sichtbarmachens reduziert werden kann, währen ein Abbild immer etwas gegenüber dem Abgebildeten Eigenständiges bleibt. Diese in der Tradition der Spiegelmetapher geläufige Reduktion

\footnotetext{
${ }^{39}$ Die Welt als Wille und Vorstellung, Bd. I (ZA Bd. 2), Anhang, S. 618.

40 Arthur Schopenhauer: Die Welt als Wille und Vorstellung. Faksimiledruck der ersten Auflage von 1819 [1818]. Frankfurt /M 1987, S. 692.
}

ethic@-Florianópolis, v. 11,n. 2,p.1-15, julho de 2012. 
auf das Leben angewandt hat zur Folge, daß das Leben als solches keine von der Bedeutung des Sichtbarmachens unabhängigen Bestimmungen haben darf. Auch die Bedingungen des Handelns, der Leib, das ihn Nährende usw. müssen in die Funktion des Spiegelns einbezogen werden. Daher kann Schopenhauer nicht beim Charakter des Menschen stehen bleiben, sondern muß nach und nach den Leib und schließlich die ganze Natur in die Deutung des Lebens als Spiegel des Willens einschließen - wie es in den Aufzeichnungen von $1814 \mathrm{zu}$ beobachten ist. Dann ist das Leben in allen seinen Aspekten - auch den naturwissenschaftlichen - nichts als Sichtbarwerden des Willens zum Leben: „Dem Willen zum Leben ist das Leben immer gewiß: denn es ist eben nichts als jener Wille selbst oder vielmehr nur sein Spiegel ... “41.

So wie dem Willen zum Leben das Leben gewiß ist, so ist umgekehrt alles Leben ein gewolltes. Darin liegt die Pointe des Schopenhauerschen Lebensbegriffs, die verloren geht, wenn man versucht, den Begriff, Willen' bei Schopenhauer durch „Kraft“, „Energie“ oder „Trieb“ zu erläutern. Vielmehr müssen umgekehrt diese Begriffe nach Schopenhauer durch den Willen erläutert werden ${ }^{42}$. Denn sie spielen zwar auf ein Inneres an, bieten aber keine Brücke, um zu ihm zu gelangen: das leistet nur das Selbstbewußtsein des bewußt wollenden Menschen, der seinem Inneren die Handlung zurechnet. So wie das innere Selbst sich im Willensakt kundtut, so tritt im Willen zum Leben das Innere der Welt ans Licht. Und so wie die Bewegung des aus dem dunklen Inneren in die Sichtbarkeit Drängenden das Entscheidende am Willensakt ist (insofern ,allererst die That" ihn als solchenerweist ${ }^{43}$ ), der zur Deutung des Willens als Ding an sich berechtigt, auch wenn es keine Erkenntnis davon geben kann: - So ist die Bewegung des Sichtbarwerdens des Willens, die Schopenhauer etwas später als „Objektivation“ bezeichnen wird, das Entscheidende am Leben.

Es ist insofern auch verfehlt, wenn Nietzsche im Zarathustra den Schopenhauerschen Willen zum Leben als „Wille zum Dasein“ dem Willen zur Macht gegenüberstellt ${ }^{44}$. Schopenhauers Wille zum Leben schließt die Tendenz zur Steigerung der Sichtbarkeit ein, die in der Stufenfolge der Ideen als Objektivationen des Willens ausgedrückt ist. Im Charakter des Menschen wird die Sichtbarkeit so groß, daß das Leben als Spiegel wahrgenommen wird und somit seine Bestimmung erfüllt. Zunächst

\footnotetext{
${ }^{41}$ HN, Bd. I, S. 166.

${ }^{42}$ Die Welt als Wille und Vorstellung, Bd. I (ZA Bd. 1), § 22, S. $156 \mathrm{f}$.

${ }^{43}$ Arthur Schopenhauer: Über die Freiheit des Willens, in: Die beiden Grundprobleme der Ethik (ZA Bd. 6). Kap. II, S. 56.

${ }^{44}$ Friedrich Nietzsche: Also sprach Zarathustra, in: KGW Bd. VI, 1, Berlin 1968, S. 144 f.
}

ethic@-Florianópolis, v. 11,n. 2, p. 1-15, julho de 2012. 
klingt diese Bestimmung noch recht Lutheranisch, indem sie mit der ,an den Früchten' zu erkennenden „Sündhaftigkeit“ verbunden wird: „Der Mensch erkennt in der Succession des Lebens, wie in einem Spiegel seinen Willen: der Schreck über diese Erkenntniß ist das Gewissen ...“45. Die Ausdehnung des Verhältnisses zwischen intelligiblem und empirischem Charakter auf den Willen und die Welt schließt das religiöse Element jedoch endgültig aus. Für Schopenhauer hängt damit auch zusammen, daß von einem Zweck des Lebens nur in uneigentlicher Rede gesprochen werden kann. Bereits 1814 steht die Philosophie Schopenhauers, die er drei Jahre später selbst in den einen Ausdruck „Die Welt ist die Selbsterkenntniß des Willens“ zusammenfaßt ${ }^{46}$, auf der Basis der Spiegelmetapher ausgebildet da. „Der Zweck des Lebens (ich brauche hier einen nur gleichnißweise wahren Ausdruck) ist die Erkenntniß des Willens. Das Leben ist der Spiegel des Willens, dessen in innrer Entzweiung bestehendes Wesen darin Objekt wird, durch welche Erkenntniß der Wille sich wenden kann und Erlösung möglich ist ${ }^{647}$. So wie von einem Zweck nur uneigentlich gesprochen werden kann, so kann auch von der „Wendung des Willens“ und von der Erlösung nur uneigentlich als einer Folge oder Wirkung der Erkenntnis die Rede sein. Der ,Zweck’ des Lebens kann kein moralischer im Sinne einer präskriptiven Ethik sein. „Es giebt kein Moralprinzip, keine Regel für das was man Wollen und nicht Wollen soll. Wie sollte es ein Gesetz geben für den Willen, der absolut frei ist? Kein absolutes Gesetz für den Willen giebt es, wohl aber einen Spiegel des Willens, der die Welt ist. Wem also die Welt, so wie sie ist, gefällt, der fahre fort, das Leben und dessen Güter zu wollen ... “48.

Die moralische Dimension der Spiegelmetapher, die nicht auf der Befolgung von Normen, sondern auf dem Bedürfnis der Erlösung beruht, ist unmittelbar aus der Verwendung der Metapher herzuleiten. Daß die Bedeutung des Lebens darin besteht, das Innere, den ,Willen' sichtbar zu machen, scheint zunächst nur auf eine Affirmation des Willens zum Leben hinauszulaufen. Es gerät nun aber auch das Subjekt des Erkennens als dasjenige in den Blick, dem etwas sichtbar gemacht wird. Wenn das Leben sich vollkommen darin erschöpft, das Sichtbarwerden des Willens - als Bewegung - für das erkennende Subjekt zu sein, dann kann dieses sich intentional entweder auf das Resultat der Bewegung, die Erscheinungen, richten oder auf das Woher der Bewegung, das als solches unzugänglich bleibt, sich aber in der Bewegung

\footnotetext{
${ }^{45}$ HN, Bd. I, S. 106.

${ }^{46}$ HN, Bd. I, S. 462.

${ }^{47}$ HN, Bd. I, S. 167.

${ }^{48}$ HN, Bd. I, S. 398.
}

ethic@-Florianópolis, v. 11, n. 2, p. 1-15, julho de 2012. 
kund tut. Im ersten Fall wird das Leben nicht in seiner Spiegelfunktion bewußt, sondern das, was der Spiegel zeigt, wird als die Sache selbst genommen. Nun ist ja das sich im Spiegel zeigende die Sache selbst, aber eben doch als Spiegelbild, das wiederum etwas anderes ist als die Sache selbst. Hier gerät die Spiegelmetapher an ihre Grenze, denn während wir normalerweise die Dinge, die wir in einem Spiegel sehen, auch außerhalb von ihm betrachten können, wird der Wille nur im Spiegel sichtbar. Eine Ausnahme, die auf das Verständnis der Metapher hinleiten kann, bildet das eigene Auge, das für uns auch nur im Spiegel sichtbar werden kann. Trotzdem wissen wir, daß es nur ein Spiegelbild ist und unterscheiden es von dem, was es zeigt. In diesem Sinne etwa ist die Umkehrung der Intention vom Leben weg zu dem sich Offenbarenden zu verstehen, die Schopenhauer als „Verneinung des Willens zum Leben“ seiner Bejahung gegenüberstellt.

Der Unterscheidung der intentionalen Ausrichtung des Blickens in den Spiegel des Lebens, die mit der nicht motivierten Bejahung und Verneinung des Willens ausgedrückt wird, korrespondiert die Unterscheidung zwischen adäquater und inadäquater Objektivation des Willens. Die Ideen sind insofern adäquate Objektivationen, als sie nicht das Resultat des Sichtbarwerdens des Willens darstellen, sondern die Bewegung selbst. Denn sie sind, wie Schopenhauer sich im Hauptwerk einmal ausdrückt, „noch nicht in [die untergeordneten Formen der Vorstellung, d.h. Raum, Zeit, Kausalität] eingegangen““49. Der Künstler „,versteht gleichsam die Natur auf halbem Worte ${ }^{\text {“50 }}$. Der gewöhnliche Mensch dagegen faßt die Resultate auf und hat es mit den inadäquaten Objektivationen zu tun. In der Kunst erfüllt sich die Bestimmung des Lebens daher am reinsten, Erkenntnis des Willens und damit dessen Beruhigung zu sein: „Wer versteht wie das Leben nichts ist als Objektivierung[,] der Spiegel des Willens, in dem er sich selbst erkennen, in sich gehen, sich beruhigen und wenden soll; der wird erst eigentlich verstehn wie die Kunst die Blüthe des Lebens sei, weil sie im wesentlichen dasselbe leistet als das Leben, aber mit Absicht und Besonnenheit, koncentrirter und vollendeter; das Leben zeigt nur Individuen, die Kunst Ideen. “51.

Die beiden Weisen der Auffassung des Spiegelbildes, die Schopenhauer auch mit dem aus der christlichen Metaphorik bekannten Gleichnis vom klaren und getrübten

\footnotetext{
${ }^{49}$ Die Welt als Wille und Vorstellung, Bd. I (ZA Bd. 1), § 32, S. 228.

${ }^{50}$ Die Welt als Wille und Vorstellung, Bd. I (ZA Bd. 1), § 45, S. 282.

${ }^{51}$ HN, Bd. I, S. 466.
} 
Spiegel umschreibt ${ }^{52}$, werden im Hauptwerk danach unterschieden, ob das Leben als Ganzes erfaßt und auf den Willen bezogen wird, oder nicht. Während der gewöhnliche Mensch nur die einzelnen Dinge, sofern sie sein gegenwärtiges Interesse tangieren, zur Kenntnis nimmt, aber nicht die Welt, und ebenso immer nur weiß, was er hier und jetzt will, aber nicht, was er überhaupt will ${ }^{53}$, schaut das künstlerische Genie in den Ideen das Leben als Ganzes, nämlich als Spiegel des einen Willens zum Leben. Dazu befähigt es ein besonderes Maß an „Besonnenheit“, und es ist nach dem bisher Gesagten durchaus vertretbar, diese ,geniale Besonnenheit““54 auf das Bewußtwerden der Spiegelfunktion des Lebens zu beziehen. So wie das Wesen des Menschen dadurch zu Tage tritt, daß die Handlungen dem Einen Subjekt des Wollens als dem sie prägenden Charakter zugeschrieben werden, so wird die Bedeutung des Lebens nur offenbar, wenn die Welt als Ganzes betrachtet wird.

$\mathrm{Da}$ der Mensch gewöhnlich weiß, was er im einzelnen Fall will und mit welchen Mitteln er seine Zwecke verfolgt; daß er aber auf die Frage, warum er es will und warum er überhaupt will und lebt, hat Clément Rosset in seinem Buch Schopenhauer, philosophe de l'absurde als die grundlegende Absurdität des Lebens bei Schopenhauer herausgestellt. Der Mensch richtet sein Leben nach Zwecken ein, aber für das Leben kann er keinen Sinn und Zweck angeben. Bei der Stelle, die Rosset als Beleg zitiert $^{55}$ - es handelt sich um den letzten Absatz des zweiten Buchs der Welt als Wille und Vorstellung - läßt er jedoch die zum dritten Buch überleitenden nachfolgenden Sätze weg. In ihnen spricht Schopenhauer von der Möglichkeit der Selbsterkenntnis des Willens im Ganzen durch die ästhetische Kontemplation: „Die einzige Selbsterkenntniß des Willens im ganzen aber ist die Vorstellung im Ganzen, die gesammte anschauliche Welt. Sie ist seine Objektität, seine Offenbarung, sein Spiegel. Was sie in dieser Eigenschaft aussagt, wird der Gegenstand unserer fernern Betrachtung seyn““56.

$\mathrm{Ob}$ allerdings die Besinnung auf die Spiegelfunktion des Lebens mit ihrer den Willen beruhigenden Konsequenz hinreicht, um das Resümee Rossets zu bestreiten, das da lautet: „Il ne faut pas compter sur le philosophe pour trouver des raisons de vivre“ ${ }^{\text {(57 }}$, will ich nicht entscheiden. Es ist jedoch zu bedenken, daß es für Schopenhauer nicht die Frage ist, ob das Leben als solches absurd ist - das steht für ihn außer Frage -, sondern

\footnotetext{
${ }^{52}$ Die Welt als Wille und Vorstellung, Bd. II (ZA Bd. 3), Kap. 22, S. 331.

${ }^{53}$ Die Welt als Wille und Vorstellung, Bd. I (ZA Bd. 1), § 29, S. 218.

${ }^{54}$ Die Welt als Wille und Vorstellung, Bd. II (ZA Bd. 4), Kap. 31, S. 452, 458.

${ }^{55}$ Clement Rosset, a.a.O., S. 74.

${ }^{56}$ Die Welt als Wille und Vorstellung, Bd. I (ZA Bd. 1), § 29, S. 218.

${ }^{57}$ Clement Rosset, a.a.O., S. 107.
} 
ob die Erkenntnis, daß das Leben absurd ist, ihm eine Bedeutung geben kann. Rosset wäre also zu fragen, ob er einen Sinn in der Aufdeckung einer Philosophie des Absurden bei Schopenhauer sieht. Denn das wäre gerade das, was der junge Schopenhauer als Ausweg aus der Mißlichkeit des Lebens ansah: den Sinn des Lebens im Nachdenken über dassel 